\title{
Quantitatively Predicting Bacterial Adhesion Using Surface Free Energy Determined with a Spectrophotometric Method
}

\author{
Xinru Zhang, ${ }^{\dagger, \ddagger}$ Qian Zhang, ${ }^{\S}$ Tao Yan, ${ }^{\S}$ Zeyi Jiang, "Xinxin Zhang," and Yi Y. Zuo*, ${ }^{*, \perp}$ \\ ${ }^{\dagger}$ Department of Mechanical Engineering, and ${ }^{\S}$ Department of Civil and Environmental Engineering, University of Hawaii at Manoa, \\ Honolulu, Hawaii 96822, United States \\ ${ }^{\ddagger}$ School of Materials Science and Engineering, and "School of Mechanical Engineering, University of Science and Technology Beijing, \\ Beijing 100083, People's Republic of China \\ ${ }^{\perp}$ Department of Pediatrics, John A. Burns School of Medicine, University of Hawaii, Honolulu, Hawaii 96826, United States
}

Supporting Information

ABSTRACT: Bacterial adhesion onto solid surfaces is of importance in a wide spectrum of problems, including environmental microbiology, biomedical research, and various industrial applications. Despite many research efforts, present thermodynamic models that rely on the evaluation of the adhesion energy are often elusive in predicting the bacterial adhesion behavior. Here, we developed a new spectrophotometric method to determine the surface free energy (SFE) of bacterial cells. The adhesion behaviors of five bacterial species, Pseudomonas putida KT2440, Salmonella Typhimurium ATCC

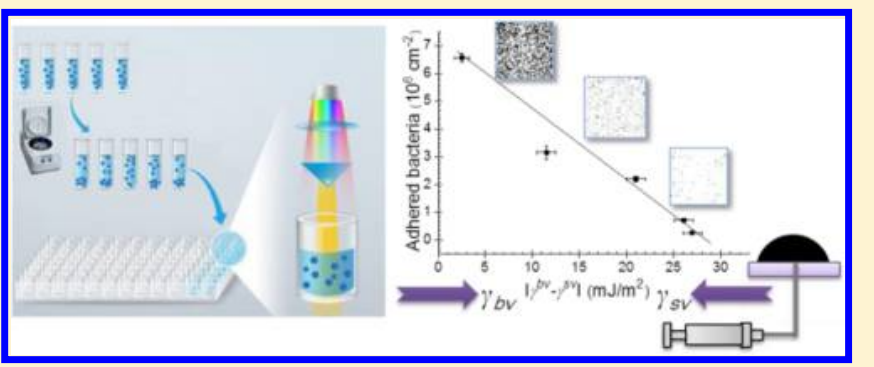
14028, Staphylococcus epidermidis ATCC 12228, Enterococcus faecalis ATCC 29212, and Escherichia coli DH5 $\alpha$, onto two model substratum surfaces, i.e., clean glass and silanized glass surfaces, were studied. We found that bacterial adhesion was unambiguously mediated by the SFE difference between the bacterial cells and the solid substratum. The lower the SFE difference, the higher degree of bacterial adhesion. We therefore propose the use of the SFE difference as an accurate and simple thermodynamic measure for quantitatively predicting bacterial adhesion. The methodological advance and thermodynamic simplification in the paper have implications in controlling bacterial adhesion and biofilm formation on solid surfaces.

\section{INTRODUCTION}

Bacterial adhesion onto solid surfaces is the onset of biofilm formation, which plays a central role in a wide spectrum of problems, including environmental microbiology, biomedical research, and various industrial applications. ${ }^{1-9}$ On the one hand, bacterial adhesion is commonly regarded as the villain in a series of adverse environmental and health issues, such as biofouling, biocorrosion, and infection of implant biomaterials and the respiratory system..$^{8-11}$ On the other hand, bacterial adhesion can be used to play a beneficial role in bioremediation of wastewater, microbial fuel cells, ${ }^{12}$ and the mineral beneficiation process. ${ }^{6,13}$ Therefore, understanding and controlling bacterial adhesion are of significant scientific and engineering interests.

Adhesion of bacteria to solid surfaces is a complex process affected by multiple factors. ${ }^{3,7,14}$ First, bacterial adhesion is influenced by physicochemical properties of the bacterial cells, such as their surface free energy (SFE) ${ }^{15-17} \zeta$ potential, ${ }^{18,19}$ production of extracellular polymeric substances, and the presence of pili and flagella. ${ }^{2,20,21}$ Second, bacterial adhesion is affected by the surface properties of substratum surfaces, including their hydrophobicity, ${ }^{8,18,22-24} \zeta$ potential, ${ }^{2,7,18}$ roughness, and microtopography. ${ }^{2,25-28}$ Third, bacterial adhesion can be also mediated by the suspending liquid medium, including its surface tension, ${ }^{29} \mathrm{pH},{ }^{30}$ ionic strength, ${ }^{31}$ temperature, and hydrodynamics. ${ }^{7,32,33}$ All of these factors affect the onset and progression of bacterial adhesion to solid surfaces.

Among all of these influencing factors, the SFEs of bacterial cells and solid surfaces have long been known to play a major role in bacterial adhesion. Neumann and co-workers developed a pioneering surface thermodynamic model to predict bacterial adhesion by evaluating the thermodynamic adhesion energy $\left(\Delta F_{\text {adh }}\right)$ between the bacterial cells and solid surfaces. ${ }^{29}$ In addition, bacterial adhesion has been studied within the scope of the classical Derjaguin-Landau-Verwey-Overbeek $(\mathrm{DLVO})^{3,14}$ and the extended DLVO (xDLVO) $)^{4,13,34}$ theories, in which the adhesion energy between bacteria and surfaces is interpreted with intermolecular and surface forces, including the van der Waals forces, electrostatic interactions, and nearsurface Lewis acid-base interactions. ${ }^{35}$

Regardless of these theoretical and experimental efforts, the existing thermodynamic models are often elusive in predicting bacterial adhesion. ${ }^{3,20,36-38}$ This is in part due to the difficulty in accurately determining the SFE of bacterial cells, which is a central parameter needed in all thermodynamic models of

Received: October 16, 2014

Revised: April 21, 2015

Accepted: April 21, 2015

Published: April 21, 2015 
bacterial adhesion. In comparison to the SFE of solid substratum surfaces, it is technically challenging to determine the SFE of bacterial cells. A common way of measuring the SFE of live cells is to first prepare a cell layer/lawn followed by Young's contact angle measurement and interpretation on this cell layer as if it was a regular ideal solid surface. ${ }^{15,18,39,40}$ The cell layer is routinely prepared by filtration, in which it is difficult to control the compactness, roughness, thickness, and even the number of filtrated cell layers. Plenty of experimental evidence showed that the contact angle measured on filtrated cell layers varies with the volume of the sessile drop, thickness, and drying time of the cell layers. ${ }^{15-17,39}$ All of these technical difficulties introduce significant methodological complication and uncertainty in determining the SFE of live cells and, hence, compromise the thermodynamic analysis of bacterial adhesion.

We recently developed a novel spectrophotometric method for directly determining the SFE of live cells. ${ }^{41,42}$ This method relies on DLVO analysis of colloidal stability and is implemented by simple spectrophotometric measurements. It is accurate, inexpensive, easy-to-use, and high-throughput. We have demonstrated its feasibility in determining the SFEs of abiotic particles, microalgae, and bacterial cells. ${ }^{41,42}$ In this paper, we revisit the classical surface thermodynamic model for bacterial adhesion, ${ }^{29}$ using the bacterial SFE determined by our new spectrophotometric method. We hypothesize that by accurately determining the bacterial SFE, the adhesion behavior of bacterial cells onto model solid surfaces can be quantitatively predicted by the difference in SFE between the bacterial cells and the solid surfaces.

\section{EXPERIMENTAL SECTION}

Thermodynamic Adhesion Energy. Similar to the prediction of liquid spreading on a solid surface by the Dupré equation, it is generally accepted that bacterial adhesion can be qualitatively predicted by the thermodynamic adhesion energy $\left(\Delta F_{\text {adh }}\right){ }^{29}$ A negative $\Delta F_{\text {adh }}$ leads to bacterial adhesion, whereas a positive $\Delta F_{\text {adh }}$ does not

$$
\Delta F_{\text {adh }}=\gamma^{\text {bs }}-\gamma^{\mathrm{bl}}-\gamma^{\text {sl }}
$$

where $\gamma^{\mathrm{bs}}, \gamma^{\mathrm{bl}}$, and $\gamma^{\mathrm{sl}}$ are the interfacial free energies of the bacteria-solid, bacteria-liquid, and solid-liquid interfaces.

These interfacial free energies $\left(\gamma^{\mathrm{bs}}, \gamma^{\mathrm{bl}}\right.$, and $\left.\gamma^{\mathrm{sl}}\right)$ can be calculated from the corresponding SFEs $\left(\gamma^{\mathrm{bv}}, \gamma^{\mathrm{sv}}\right.$, and $\left.\gamma^{\mathrm{lv}}\right)$ via appropriate combining rules. ${ }^{39,40}$ Without the loss of generality, using Neumann's equation of state, ${ }^{43} \gamma^{\mathrm{bs}}, \gamma^{\mathrm{bl}}$, and $\gamma^{\mathrm{sl}}$ can be expressed as

$$
\begin{aligned}
& \gamma^{\mathrm{bs}}=\gamma^{\mathrm{bv}}+\gamma^{\mathrm{sv}}-2 \sqrt{\gamma^{\mathrm{bv}} \gamma^{\mathrm{sv}}} \mathrm{e}^{-\beta\left(\gamma^{\mathrm{bv}}-\gamma^{\mathrm{sv}}\right)^{2}} \\
& \gamma^{\mathrm{bl}}=\gamma^{\mathrm{bv}}+\gamma^{\mathrm{lv}}-2 \sqrt{\gamma^{\mathrm{bv}} \gamma^{\mathrm{lv}}} \mathrm{e}^{-\beta\left(\gamma^{\mathrm{bv}}-\gamma^{\mathrm{lv}}\right)^{2}} \\
& \gamma^{\mathrm{sl}}=\gamma^{\mathrm{sv}}+\gamma^{\mathrm{lv}}-2 \sqrt{\gamma^{\mathrm{sv}} \gamma^{\mathrm{lv}}} \mathrm{e}^{-\beta\left(\gamma^{\mathrm{sv}}-\gamma^{\mathrm{lv}}\right)^{2}}
\end{aligned}
$$

where the value of $\beta$ was experimentally determined to be $0.0001247\left(\mathrm{~mJ} / \mathrm{m}^{2}\right)^{-2} \cdot{ }^{43}$

Substituting eqs $2-4$ into eq 1 yields

$$
\begin{aligned}
\Delta F_{\text {adh }}= & 2 \sqrt{\gamma^{\text {bv }} \gamma^{\text {lv }}} \mathrm{e}^{-\beta\left(\gamma^{\text {bv }}-\gamma^{\text {lv }}\right)^{2}}+2 \sqrt{\gamma^{\text {sv }} \gamma^{\text {lv }}} \mathrm{e}^{-\beta\left(\gamma^{\text {sv }}-\gamma^{\text {lv }}\right)^{2}} \\
& -2 \sqrt{\gamma^{\text {bv }} \gamma^{\text {sv }}} \mathrm{e}^{-\beta\left(\gamma^{\text {bv }}-\gamma^{\text {sv }}\right)^{2}}-2 \gamma^{\text {lv }}
\end{aligned}
$$

With eq 5 , the adhesion energy $\left(\Delta F_{\text {adh }}\right)$ can be estimated by measuring SFEs of the bacterial cells $\left(\gamma^{\mathrm{bv}}\right)$, the solid substratum $\left(\gamma^{\mathrm{sv}}\right)$, and the liquid medium $\left(\gamma^{\mathrm{lv}}\right)$ (note that the superscript $\mathrm{v}$ represents the vapor phase in which the SFE is determined). It can be derived from eq 5 that, within the same liquid medium (i.e., constant $\gamma^{\mathrm{lv}}$ ), the smaller the SFE difference between the bacterial cells and the solid surface (i.e., $\left|\gamma^{\mathrm{bv}}-\gamma^{\mathrm{sv}}\right|$ ), the more negative the adhesion energy and, hence, more bacterial adhesion is expected. In other words, the degree of bacterial adhesion should increase with diminishing energy difference between the bacterial cells and the substratum surface. We will test this hypothesis in the following experiments.

Bacteria Culture and Preparation of Substratum Surfaces. We focused on five widely studied bacterial species, i.e., Pseudomonas putida KT2440, Salmonella Typhimurium ATCC 14028, Staphylococcus epidermidis ATCC 12228, Enterococcus faecalis ATCC 29212, and Escherichia coli DH5 $\alpha$ (see Table S1 of the Supporting Information for a summary of morphological and surface properties of these bacterial cells). All bacteria except $S$. epidermidis were cultured in Luria-Bertani (LB) broth. S. epidermidis was cultured in a nutrient broth consisting of $10 \mathrm{~g}$ of tryptone, $3 \mathrm{~g}$ of beef extract, and $5 \mathrm{~g}$ of $\mathrm{NaCl}$ in $1 \mathrm{~L}$ of water. All bacteria were harvested at the stationary phase $\left(\mathrm{OD}_{600}\right.$ of approximately 2$)$. The cultivated bacteria suspensions were centrifuged at $3600 \mathrm{~g}$ for $3 \mathrm{~min}$ to remove cell debris, followed by three rounds of centrifugation and washing with phosphate buffer solutions (PBS). The bacteria cells were then resuspended in fresh culture medium, followed by $1 \mathrm{~min}$ vortex and $1 \mathrm{~min}$ sonication, to form homogeneous cell suspensions at concentrations of approximately $10^{10}$ cells $/ \mathrm{mL}$ (for determining the SFE) and $10^{8}$ cells/ $\mathrm{mL}$ (for studying bacterial adhesion).

Two types of solid surfaces were prepared as the model substratum surfaces. Thoroughly cleaned microscopy glass slides (Millicell EZ SLIDE, Millipore, Germany) were used as a representative hydrophilic surface. Silanized glass slides were used as a representative hydrophobic surface. For glass silanization, microscopy glass slides were first cleaned with acetone and dried under nitrogen flow. The cleaned glass slides were then placed in a glass Petri dish at $70{ }^{\circ} \mathrm{C}$ for $12 \mathrm{~h}$ to allow for reaction with the vapor of 1,1,1,3,3,3-hexamethyldisilazane (HMDS, SPI Supplies, West Chester, PA).

Determination of SFEs of Cultural Medium $\left(\gamma^{\text {Iv }}\right)$, Substratum Surface $\left(\gamma^{\text {sv }}\right)$, and Bacterial Cells $\left(\gamma^{\text {bv }}\right)$. $\gamma^{\text {lv }}$ of the cultural medium was determined with the pendant drop method using axisymmetric drop shape analysis (ADSA). ${ }^{44} \gamma^{\mathrm{lv}}$ of the LB broth was measured to be $62.0 \pm 0.2 \mathrm{~mJ} / \mathrm{m}^{2}$. $\gamma^{\mathrm{lv}}$ of the culture medium for $S$. epidermidis was measured to be 57.8 $\pm 0.2 \mathrm{~mJ} / \mathrm{m}^{2} \cdot \gamma^{\mathrm{sv}}$ was determined with the low-rate dynamic contact angle method in conjunction with Neumann's equation of state. ${ }^{43}$ A detailed description of the low-rate dynamic contact angle method and intermediate experimental results can be found in the Supporting Information. $\gamma^{\mathrm{sv}}$ of the clean glass surface and the silanized glass surface was determined to be $70 \pm 1$ and $38 \pm 1 \mathrm{~mJ} / \mathrm{m}^{2}$, respectively. These measurements are in good agreement with literature values. ${ }^{45}$

$\gamma^{\text {bv }}$ was determined using our new spectrophotometric method. A detailed description of the spectrophotometric method can be found elsewhere. ${ }^{41,42}$ Principles of this method are based on the analysis of colloidal stability of cell suspensions. According to the classical DLVO theory, the colloidal stability of a cell suspension depends upon the balance between van der Waals attraction and electrostatic repulsion. A minimum van der Waals attraction can be obtained by dispersing cells in a liquid medium of which surface tension 


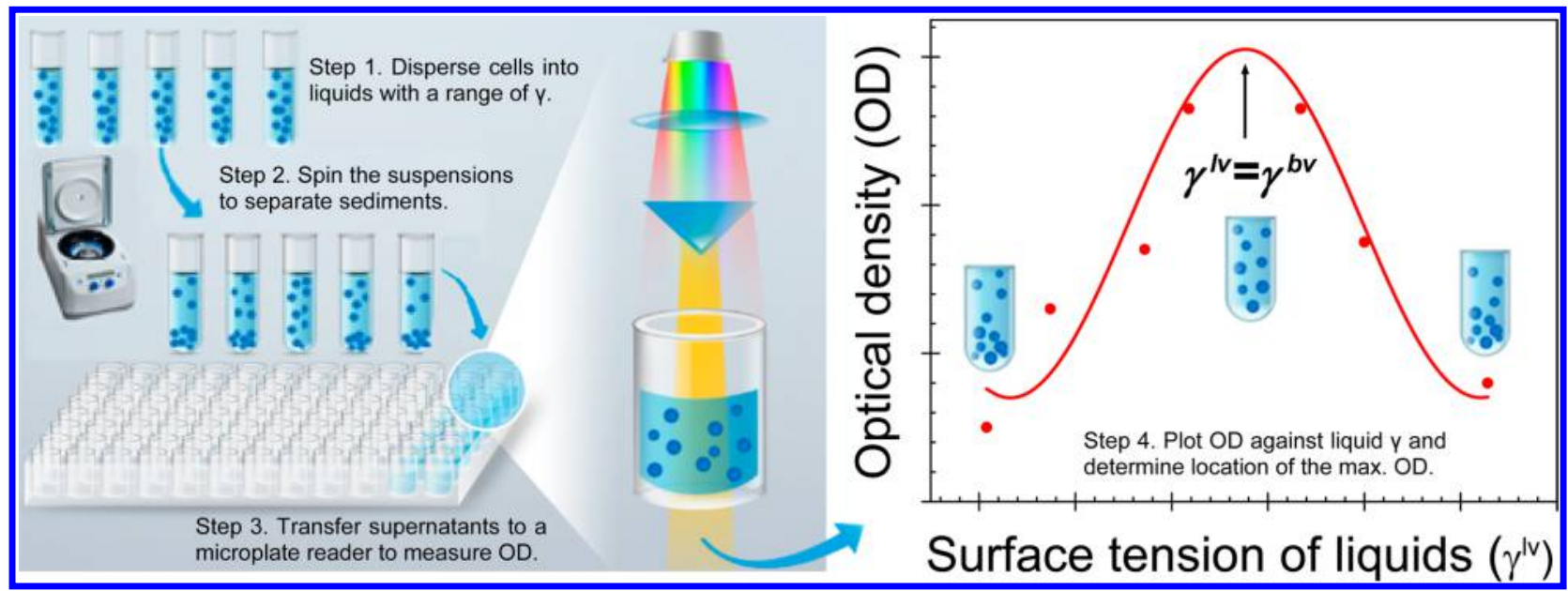

Figure 1. Schematic of the spectrophotometric method for determining the SFE of bacterial cells $\left(\gamma^{\mathrm{bv}}\right)$. First, bacterial cells were dispersed into liquid media with a range of surface tensions $\left(\gamma^{\mathrm{lv}}\right)$. Second, the cell suspensions were centrifuged to separate the supernatant from the sediment. Third, the series of supernatants were transferred to a 96 well microplate. The degree of cell dispersion in these supernatants was compared by measuring their OD. Finally, OD of these supernatants was plotted against $\gamma^{\mathrm{lv}}$. The location of the maximum OD, at which $\gamma^{\mathrm{bv}}=\gamma^{\mathrm{lv}}$, was determined with polynomial fitting.

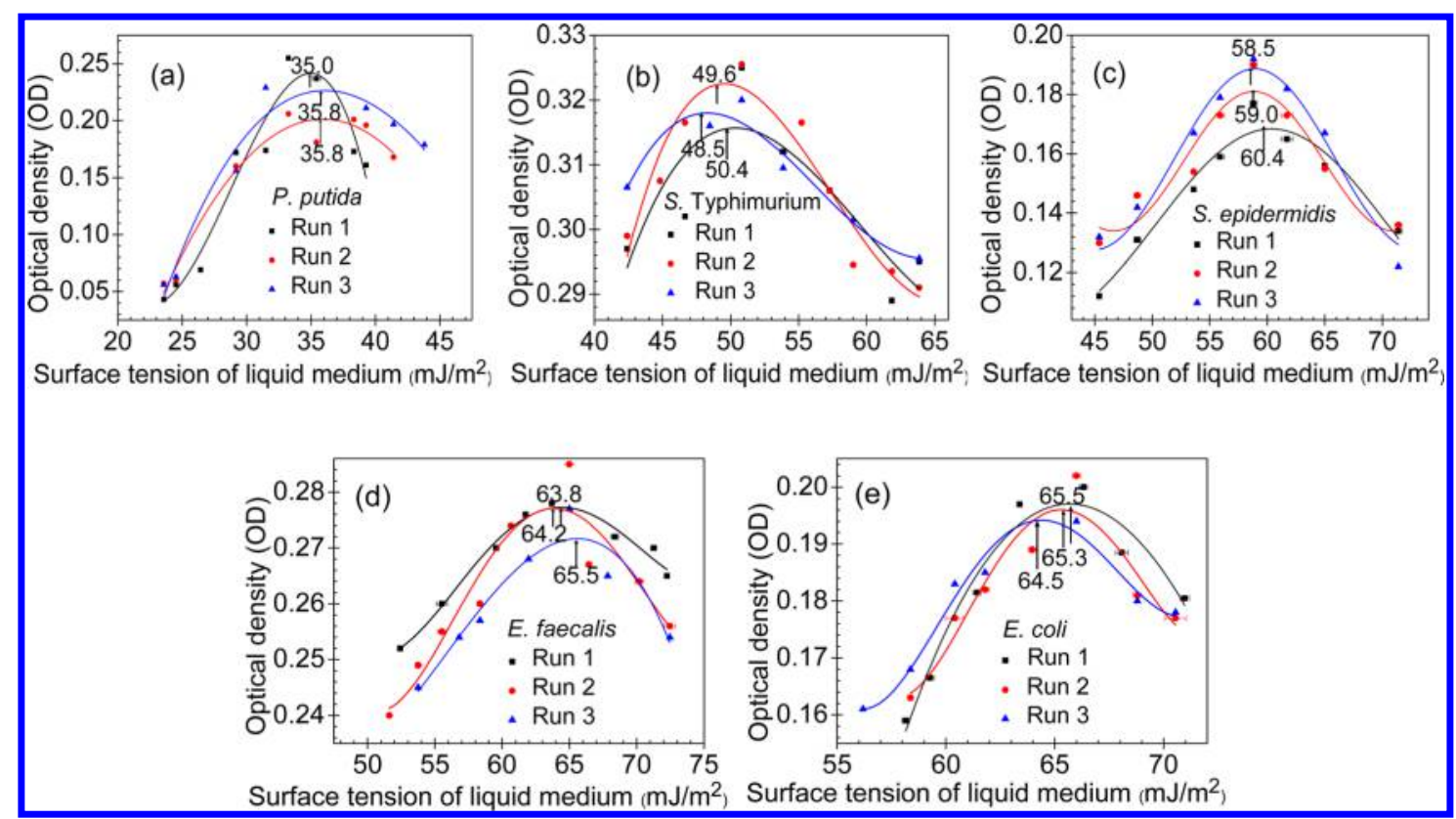

Figure 2. Determination of the SFE of five bacterial cell species using the spectrophotometric method: (a) P. putida, (b) Salmonella Typhimurium, (c) S. epidermidis, (d) E. faecalis, and (e) E. coli. For each bacterial species, three individual experimental runs are presented to show the repeatability. Locations of the OD peaks correspond to the SFEs of these bacterial cells.

is close to the SFE of the cells, at which the cells are expected to disperse in the medium without significant aggregation and sedimentation. Hence, when dispersing cells in a series of liquids with known surface tension values, surface tension of the liquid with the maximum level of cell dispersion should be equal to the SFE of the cells. After removal of large aggregates with appropriate centrifugation (described in detail below), the degree of cell dispersion in these liquids can be compared by measuring the optical density (OD) of the suspension. The highest degree of cell dispersion corresponds to a maximum OD. Consequently, the surface tension of the liquid in which the maximum OD appears should be close to the SFE of the cells.

Implementation of the spectrophotometric method is illustrated in Figure 1. A series of liquid media with surface tensions ranging from 22 to $72 \mathrm{~mJ} / \mathrm{m}^{2}$, each at $0.5 \mathrm{~mL}$, were prepared with binary mixtures of ethanol and water at predefined mixing ratios. Surface tensions of these liquids were quantified by the pendant drop method. A total of $10 \mu \mathrm{L}$ bacterial suspension with a concentration of approximately $10^{10}$ cells $/ \mathrm{mL}$ was added to each liquid medium, followed by vortex mixing and leaving undisturbed for $20 \mathrm{~min}$. These liquid media were subsequently centrifuged at $100 \mathrm{~g}$ for $6 \mathrm{~min}$ to separate the supernatant from the sediment. This centrifugation speed was carefully selected to be high enough to spin down aggregated cells but low enough to keep individually dispersed cells in the supernatant. We found nearly no separation happening in the liquid medium of which surface tension is close to the SFE of the bacterial cells. In this liquid medium, cells were uniformly dispersed without significant aggregation. A total of $200 \mu \mathrm{L}$ of 
supernatant of each liquid medium was then transferred to a 96 well microplate. OD at the wavelength of $600 \mathrm{~nm}\left(\mathrm{OD}_{600}\right)$ was measured by a microplate reader (Epoch, BioTek, Winooski, $\mathrm{VT}) . \mathrm{OD}_{600}$ was plotted against surface tensions of the liquid media, and peaks were determined by the third-order polynomial fitting. All measurements were performed at least 3 times and averaged.

Bacterial Adhesion Experiments. Bacterial adhesion was studied using a glass cell analysis chamber (Millicell EZ SLIDE), with the clean microscopy glass or the silanized glass slide as the substratum. A total of $1 \mathrm{~mL}$ of bacterial cell suspension at a concentration of $10^{8}$ cells $/ \mathrm{mL}$ was added to each chamber and incubated at $37{ }^{\circ} \mathrm{C}$ for $2 \mathrm{~h}$. After incubation, the substratum was removed from the chamber, gently rinsed with PBS to remove non-adherent bacteria, and air-dried. Cells adherent to the substratum were stained by methylene blue and numerated by an optical microscope (BX51, Olympus, Japan). The number of adhered bacterial cells was analyzed using ImageJ [National Institutes of Health (NIH)]. All measurements were performed at least 3 times, and results were shown in mean \pm standard deviation (SD).

\section{RESULTS AND DISCUSSION}

SFE of Bacterial Cells $\left(\gamma^{\mathrm{bv}}\right)$. Figure 2 shows the experimental results of measuring the SFE of five bacterial species, P. putida (a), Salmonella Typhimurium (b), S. epidermidis (c), E. faecalis (d), and E. coli (e), using the spectrophotometric method. For each bacterial species, three experimental runs are presented to show the repeatability of our measurements. It can be seen that $\mathrm{OD}_{600}$ measured from different experimental runs notably scatters. This is due to variations in the total number of cells when dispersing a small amount $(10 \mu \mathrm{L})$ of highly concentrated $\left(10^{10}\right.$ cells $\left./ \mathrm{mL}\right)$ cell suspensions in each experimental run. Despite scattering between experimental runs, the peak OD values determined from different experimental runs collapse into the same surface tension range for each bacteria studied. Locations of these OD peaks, indicating the maximum degree of cell dispersion, correspond to the characteristic SFEs of these bacterial cells, i.e., $35.5 \pm 0.5,49.5 \pm 0.9,59.3 \pm 0.9,64.5 \pm 0.9$, and $65.1 \pm$ $0.5 \mathrm{~mJ} / \mathrm{m}^{2}$, respectively. These results are consistent with our previous measurements, in which the characteristic SFEs of various bacterial species were used for cell sorting. ${ }^{42}$

Because of the overabundance of bacterial species and strains, we can only conduct a limited direct comparison between our measurements and those reported by others. Using contact angle measurement in conjunction with Neumann's equation of state, Sharma and Rao reported the SFE of E. faecalis ATCC 29212 to be $64.45 \mathrm{~mJ} / \mathrm{m}^{2}$. ${ }^{39}$ This is in excellent agreement with our spectrophotometric measurement of the same bacterial cells, i.e., $64.5 \mathrm{~mJ} / \mathrm{m}^{2}$. The SFE of E. coli of various strains was reported to be around $66-67 \mathrm{~mJ} / \mathrm{m}^{2}, 15,29,39$ which is also in good agreement with our measurements, i.e., $65.1 \mathrm{~mJ} / \mathrm{m}^{2}$.

To further verify the accuracy of our spectrophotometric method, we have determined the SFE of these bacterial cells by carefully performing the classical contact angle method. ${ }^{29,40}$ Experimental details and results can be found in the Supporting Information. We found an excellent agreement between these two methods with a general discrepancy of less than $1 \mathrm{~mJ} / \mathrm{m}^{2}$ for the measured SFEs of bacterial cells. However, in comparison to the contact angle method, which involves measuring the low-rate dynamic contact angle (see Figure S3 of the Supporting Information) and theoretical interpretation using Neumann's equation of state (see Table S3 of the Supporting Information), the spectrophotometric method is much simpler, quicker, and less dependent upon the skills of the operator.

Bacterial Adhesion to Substratum Surfaces. Figure 3 shows the images of bacterial adhesion onto clean glass surfaces

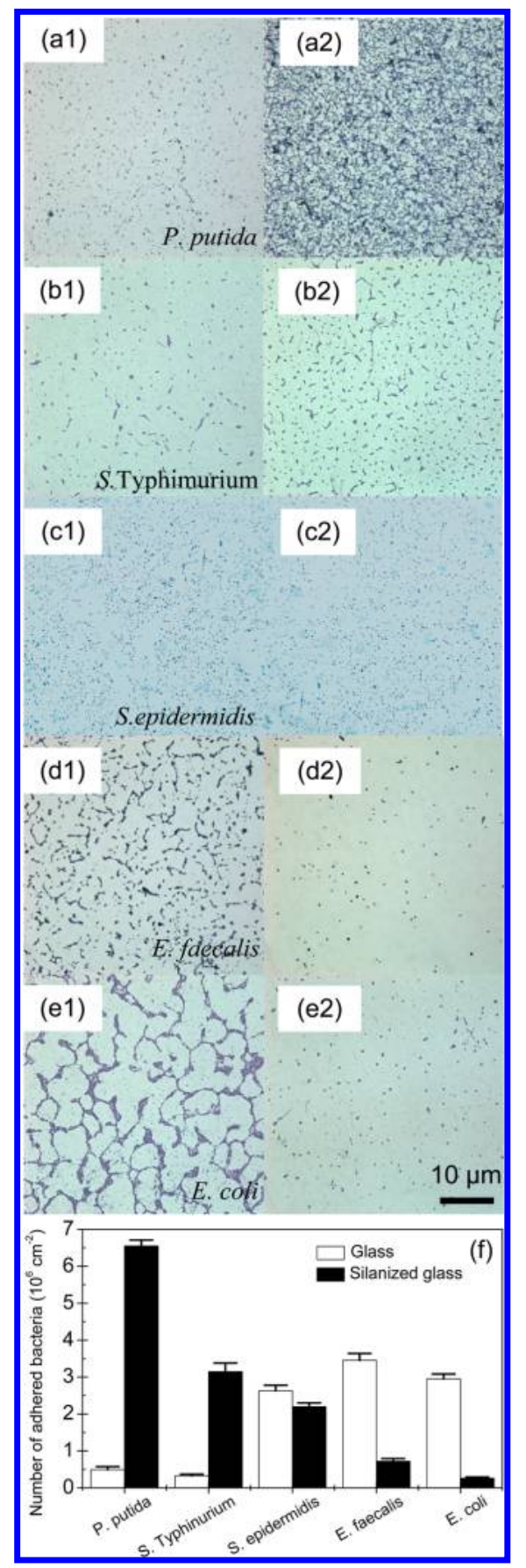

Figure 3. Images of bacterial adhesion onto clean glass surfaces (a1e1) and silanized glass surfaces (a2-e2). The five bacterial species are P. putida (a), SalmonellaTyphimurium (b), S. epidermidis (c), E. faecalis (d), and E. coli (e). (f) Number of adhered bacterial cells per unit surface area in the above cases. It appears that the high-SFE bacteria cells, E. faecalis (d1) and E. coli (e1), preferentially adhere to the highSFE clean glass surfaces, while the low-SFE bacterial cells, $P$. putida (a2) and Salmonella Typhimurium (b2), selectively adhere to the lowSFE silanized glass surfaces. 


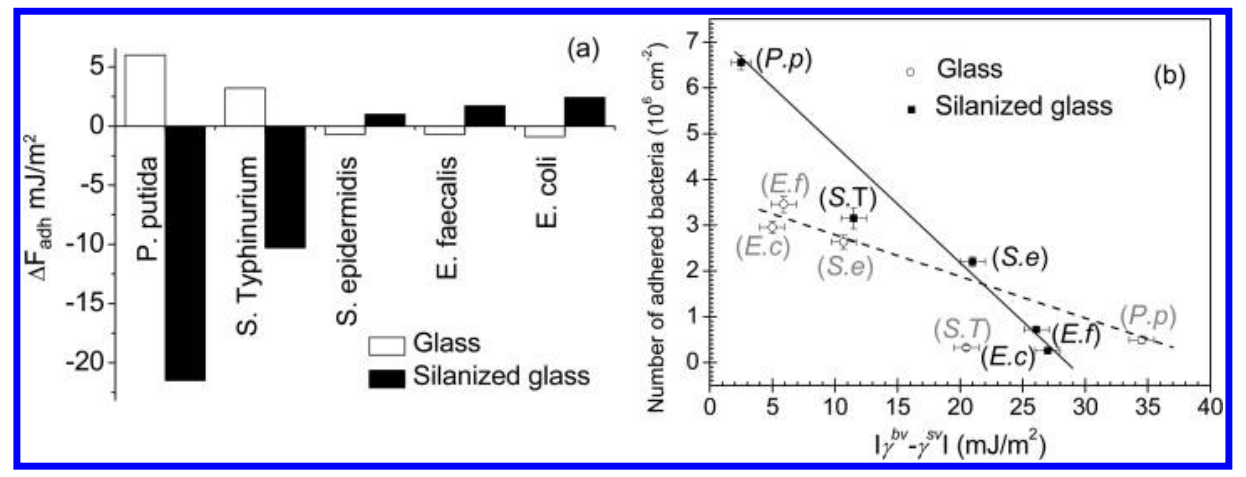

Figure 4. Bacterial adhesion correlated with (a) adhesion energy $\left(\Delta F_{\text {adh }}\right)$ and (b) SFE difference between $\gamma^{\mathrm{bv}}$ and $\gamma^{\mathrm{sv}}$, i.e., $\mid \gamma^{\mathrm{bv}}-\gamma^{\mathrm{sv}}$. Abbreviations used in panel b are P.p, P. putida; S.T, Salmonella Typhimurium; S.e, S. epidermidis; E.f, E. faecalis; and E.c, E. coli.

(left column) and onto silanized glass surfaces (right column). Figure $3 f$ shows the number of adhered bacterial cells per unit surface area on these solid substrata. It appears that the highSFE bacteria cells, E. faecalis (Figure 3d1) and E. coli (Figure $3 \mathrm{e} 1$ ), preferentially adhere to the high-SFE (i.e., hydrophilic) clean glass surfaces, while the low-SFE bacterial cells, $P$. putida (Figure 3a2) and Salmonella Typhimurium (Figure 3b2), preferentially adhere to the low-SFE (i.e., hydrophobic) silanized glass surfaces.

Surface Thermodynamics of Bacterial Adhesion. To better understand the surface thermodynamics of bacterial adhesion, we calculated the adhesion energy $\left(\Delta F_{\text {adh }}\right)$ using eq 5 based on our experimentally determined SFEs $\left(\gamma^{\mathrm{bv}}, \gamma^{\mathrm{sv}}\right.$, and $\left.\gamma^{\mathrm{lv}}\right)$. The sign of $\Delta F_{\text {adh }}$ has long been used as a qualitative criterion to predict bacterial adhesion: a negative $\Delta F_{\text {adh }}$ leads to bacterial adhesion, whereas a positive $\Delta F_{\text {adh }}$ does not. ${ }^{3,19,29,36}$ Figure $4 \mathrm{a}$ shows $\Delta F_{\text {adh }}$ calculated for all bacteria-surface pairs shown in Figure 3. Among all 10 bacteria-surface pairs studied, $\Delta F_{\text {adh }}$ is negative for P. putida (Figure 3a2) and Salmonella Typhimurium (Figure $3 \mathrm{~b} 2$ ) on silanized glass surfaces and S. epidermidis (Figure 3c1), E. faecalis (Figure 3d1), and E. coli (Figure 3e1) on clean glass surfaces, indicating thermodynamic conditions favorable for bacterial adhesion. $\Delta F_{\text {adh }}$ is positive for the other five bacteria-surface pairs, indicating unfavorable conditions. It appears that the sign of $\Delta F_{\text {adh }}$ generally agrees with the behavior of bacterial adhesion (Figure 3f). However, when the absolute value of $\Delta F_{\text {adh }}$ is small (i.e., close to zero), such as in the case of $S$. epidermidis adhering to clean or silanized glass surfaces, the sign of $\Delta F_{\text {adh }}$ fails to predict the behavior of bacterial adhesion (Figure $3 \mathrm{c} 1$ versus Figure $3 \mathrm{c} 2$ ).

The sign of $\Delta F_{\text {adh }}$ can be only used to qualitatively predict the behavior of bacterial adhesion but not for quantitative analysis. ${ }^{1,3,36,37}$ By analyzing eq 5, we found that the quantity of $\Delta F_{\text {adh }}$ appears to correlate with the SFE difference between the bacterial cells and the solid substratum $\left|\gamma^{\mathrm{bv}}-\gamma^{\mathrm{sv}}\right|$. In Figure $4 \mathrm{~b}$, we plot the number of adhered bacterial cells as a function of the bacterial-substratum SFE difference. It is clear that, regardless of the specific bacterial species and substratum surfaces (hydrophilic or hydrophobic), the degree of bacterial adhesion nearly linearly increases with decreasing SFE difference, i.e., diminishing energy difference between the bacterial cells and the substratum surface. In comparison to the sign of the adhesion energy $\Delta F_{\text {adh }}$ commonly used in predicting bacterial adhesion, we hereby propose the use of the bacteriasubstratum SFE difference $\left|\gamma^{\mathrm{bv}}-\gamma^{\mathrm{sv}}\right|$ to quantitatively predict bacterial adhesion.
Up to now, we have limited our discussion into the absolute value of $\left|\gamma^{\text {bv }}-\gamma^{\mathrm{sv}}\right|$. As shown in Figure $4 \mathrm{~b}$, we found that $\mid \gamma^{\text {bv }}-$ $\gamma^{\mathrm{sv}}$ can be used as a simple criterion for predicting adhesion of different bacteria onto a hydrophobic surface (guided by the solid line) or onto a hydrophilic surface (guided by the dashed line). Similarly, $\left|\gamma^{\mathrm{bv}}-\gamma^{\mathrm{sv}}\right|$ can be used to predict adhesion of one bacterial species onto either a hydrophobic or hydrophilic surface. However, Figure $4 \mathrm{~b}$ also shows that, even with a similar $\left|\gamma^{\mathrm{bv}}-\gamma^{\mathrm{sv}}\right|$, the extent of different bacteria attaching to different surfaces can vary significantly. For example, although $\left|\gamma^{\text {bv }}-\gamma^{s v}\right|$ is close between P. putida at the silanized glass and E. coli at the unmodified glass, the extent of bacterial adhesion in these two cases varies by a factor more than 2 . This indicates that the absolute value of $\left|\gamma^{\mathrm{bv}}-\gamma^{\mathrm{sv}}\right|$ cannot be the only factor in determining the general pattern of bacterial adhesion. Without detailed investigation, it is interesting to point out that, for all bacterial species studied on the silanized glass surface $\left(\gamma^{\mathrm{sv}}=38\right.$ $\left.\mathrm{mJ} / \mathrm{m}^{2}\right)$, P. putida $\left(\gamma^{\mathrm{bv}}=35.5 \mathrm{~mJ} / \mathrm{m}^{2}\right)$ is the only species of which $\gamma^{\text {bv }}<\gamma^{\text {sv }}$, i.e., with a negative sign of $\left(\gamma^{\text {bv }}-\gamma^{\text {sv }}\right)$. This may indicate that the sign of $\left(\gamma^{\mathrm{bv}}-\gamma^{\mathrm{sv}}\right)$, in addition to its absolute value, is also meaningful in affecting bacterial adhesion.

Implications in Cell Surface Hydrophobicity. Bacterial adhesion is the initial step of biofilm formation on any biotic or abiotic surface. ${ }^{46}$ The driving forces for the freely suspended planktonic organisms to attach to a solid surface appear to be complex and are affected by multiple factors. Numerous studies have demonstrated that the bacterial cell surface hydrophobicity (CSH) plays an important role in the initial attachment of bacteria to solid surfaces. ${ }^{46,47}$ Despite the intrinsic heterogeneity of cell surfaces, the bacterial $\mathrm{CSH}$ reflects the overall contribution of molecular and topographic structures to the hydrophobic properties of microbial surfaces. It is found that bacteria with higher $\mathrm{CSH}$ have a higher tendency to attach to hydrocarbons and adhere more to hydrophobic nonpolar surfaces. ${ }^{47}$ A series of techniques have been developed to measure bacterial $\mathrm{CSH}$. The most popular techniques rely on the measurement of microbial adhesion to hydrocarbons (MATH). ${ }^{48}$ The MATH-like assays compare the preference of bacteria to the aqueous phase and to a hydrocarbon phase or hydrophobic ligands. Hence, MATH only measures the relative $\mathrm{CSH}$. MATH results from the literature can be hardly compared directly because of the different test conditions used in different studies. ${ }^{49}$ More recent studies use alternative measures to quantify $\mathrm{CSH}$, such as the contact angle measured on bacterial cell lawns ${ }^{15}$ and interfacial rheology measured at bacteria-adsorbed oil-water interfaces. ${ }^{49}$ 
In this study, we have demonstrated the use of SFE as a quantitative measure of $\mathrm{CSH}$. We found increasing SFE of the five bacterial species used in this study, i.e., P. putida $(35.5 \mathrm{~mJ} /$ $\left.\mathrm{m}^{2}\right)<$ Salmonella Typhimurium $\left(49.5 \mathrm{~mJ} / \mathrm{m}^{2}\right)<$ S. epidermidis $\left(59.3 \mathrm{~mJ} / \mathrm{m}^{2}\right)<$ E. faecalis $\left(64.5 \mathrm{~mJ} / \mathrm{m}^{2}\right)<$ E. coli $\left(65.1 \mathrm{~mJ} / \mathrm{m}^{2}\right)$, indicating a decrease in CSH of these bacteria. The ranking of $\mathrm{CSH}$ determined with the SFE in our study is in good agreement with the ranking determined by Rühs et al. using the MATH method and their interfacial tension measurements, i.e., CSH of P. putida > Salmonella Typhimurium > E. coli. ${ }^{49}$ Therefore, the development of our easy-to-use spectrophotometric method not only provides a practical means of determining the SFE of live cells but also creates a novel method of quantitatively determining the microbial CSH.

Implications in Biofilm Formation. Despite the many years of research efforts, the underlying mechanism behind bacterial adhesion is unknown. Considerable discrepancy exists in the literature when attempting to generalize bacterial adhesion behavior from phenomenological measurements with individual bacteria and surfaces. On the one hand, as summarized in Donlan's seminal review, ${ }^{7}$ many researchers found that bacteria attach more rapidly to hydrophobic nonpolar surfaces, such as plastics, than to hydrophilic surfaces, such as glass or metals. On the other hand, extensive research evidence shows that bacteria (mainly those related to foodprocessing surfaces, such as Listeria monocytogenes) attach more to high-energy hydrophilic surfaces, including stainless steel and glass, than to hydrophobic polymeric surfaces. ${ }^{50}$

Our present study suggests that the actual behavior of bacterial adhesion is governed by the hydrophobicity (or SFE) of not only the colonized solid surfaces but also the bacterial cells. For the solid surface, its apparent hydrophobicity is affected by both roughness and physicochemical properties, mainly the SFE. For an ideal surface (smooth and homogeneous), the SFE is a measure of the intrinsic surface hydrophobicity determined by the chemical structure and intermolecular forces of the surface. The lower the SFE, the more hydrophobic the surface. For real surfaces, however, roughness usually contributes to the apparent surface hydrophobicity. Interrelations between hydrophobicity and roughness in real surfaces complicate energetic interpretation of bacterial adhesion. Here, we circumvented this difficulty by studying bacterial adhesion onto two smooth model surfaces, i.e., unmodified and silanized glass surfaces. Their differences in surface hydrophobicity are purely due to their different SFEs determined with the low-rate dynamic contact angle method in conjunction with Neumann's equation of state approach, i.e., the SFE of $70 \mathrm{~mJ} / \mathrm{m}^{2}$ for unmodified glass versus $38 \mathrm{~mJ} / \mathrm{m}^{2}$ for silanized glass.

As for the selection of bacteria, not only do these bacterial species show a gradient of CSH (or SFE), but they are also representative for biofilm formation under various environmental and clinical conditions. Among the three Gram-negative bacterial species used here, E. coli is perhaps the most wellstudied model organism in microbiology. Salmonella Typhimurium is a common pathogenic microbe found in foodborne biofilms. P. putida is a plant-associated bacterium often found in soil biofilms and most studied for bioremediation and wastewater treatment. The two Gram-positive bacteria used in this study, i.e., S. epidermidis and E. faecalis, are most studied for biofilm formation related to nosocomial infections. Both $S$. epidermidis and E. faecalis have been found in biofilms developed on indwelling medical devices, implants, and living tissues, such as the central venous catheter, urinary catheter, prosthetic heart valve, and artificial voice prosthesis. ${ }^{51}$

After carefully determining SFEs of the model substratum surfaces and the bacterial cells, we found that the extent of bacterial adhesion can be quantitatively correlated with the SFE difference between the bacterial cells and the substrata, i.e., $\mid \gamma^{\text {bv }}$ $-\gamma^{\mathrm{sv}}$. This finding is important because it suggests that bacterial adhesion, at least the initial attachment, is driven by a pure energetic potential. Instead of simply stating that bacteria prefer to attach to a hydrophobic or hydrophilic surface, our study provides a more general criterion for quantitatively predicting bacterial adhesion. We argue that bacteria prefer to attach to solid surfaces of similar SFE or hydrophobicity. In other words, the smaller the SFE difference between the bacteria and the solid surface $\left|\gamma^{\mathrm{bv}}-\gamma^{\mathrm{sv}}\right|$, the more adhesion is expected. It should be emphasized that feasibility of using the SFE difference as a practical criterion to evaluate bacterial adhesion stems from our novel high-throughput spectrophotometric method for determining the bacterial SFE, which can be easily operated in any lab with a microplate reader.

Cautions, however, should be exercised when generalizing our findings to biofilm formation because our experiments were conducted under strictly controlled laboratory conditions. Microbial colonization on real surfaces is much more complicated than on our model substrata because the apparent hydrophobicity of a real surface is affected by its roughness and heterogeneity. More importantly, the bacterial CSH has been shown to be affected by the microbial growth rate, growth medium, and culture conditions. ${ }^{50}$ Hence, the bacterial CSH can change dramatically at different stages of cell dividing and in response to changes in the environment. ${ }^{46}$ All of these factors may introduce deviations from our energetic interpretation of bacterial adhesion.

In summary, we have developed a novel high-throughput spectrophotometric method that allows us to accurately determine the SFE of live bacterial cells. Using this method, we have studied the adhesion behavior of five bacterial species onto two model substratum surfaces. We showed that the SFE difference between the bacterial cells and the solid substratum, i.e., $\left|\gamma^{\mathrm{bv}}-\gamma^{\mathrm{sv}}\right|$, can be used as an accurate and simple thermodynamic criterion for quantitatively predicting bacterial adhesion. The methodological advance and thermodynamic simplification have scientific and engineering implications in controlling bacterial adhesion and biofilm formation on solid surfaces.

\section{ASSOCIATED CONTENT}

\section{Supporting Information}

Summary of morphological and surface properties for bacterial cells used in this study (Table S1), schematic of the low-rate dynamic contact angle method (Figure S1), representative experimental results (i.e., contact angle, contact radius, and drop volume) for low-rate dynamic contact angle measurements on a clean glass surface (a) and a silanized glass surface (b) (Figure S2), results for determining $\gamma^{\mathrm{sv}}$ of clean glass and silanized glass surfaces (Table S2), representative experimental results (i.e., contact angle, contact radius, and drop volume) for low-rate dynamic contact angle measurements on carefully prepared bacteria lawns: (a) P. putida, (b) Salmonella Typhimurium, (c) S. epidermidis, (d) E. faecalis, and (e) E. coli (Figure S3), comparison of the SFE of bacterial cells $\left(\gamma^{\mathrm{bv}}\right)$ determined with the classical contact angle method and the spectrophotometric method (Table S3), and results for 
determining the adhesion energy $\left(\Delta F_{\text {adh }}\right)$ of bacterial cells on the clean glass surface $\left(\gamma^{\mathrm{sv}}=70 \mathrm{~mJ} / \mathrm{m}^{2}\right)$ and the silanized glass surfaces $\left(\gamma^{\mathrm{sv}}=38 \mathrm{~mJ} / \mathrm{m}^{2}\right)$ (Table S4). The Supporting Information is available free of charge on the ACS Publications website at DOI: $10.1021 /$ es5050425.

\section{AUTHOR INFORMATION}

\section{Corresponding Author}

*Telephone: 808-956-9650. Fax: 808-956-2373. E-mail: yzuo@ hawaii.edu.

Notes

The authors declare no competing financial interest.

\section{ACKNOWLEDGMENTS}

This work was supported by National Science Foundation (NSF) Grants CBET-1254795 (to Yi Y. Zuo) and CBET1236596 (to Yi Y. Zuo).

\section{REFERENCES}

(1) Hori, K.; Matsumoto, S. Bacterial adhesion: From mechanism to control. Biochem. Eng. J. 2010, 48 (3), 424-434.

(2) Tuson, H. H.; Weibel, D. B. Bacteria-surface interactions. Soft Matter 2013, 9 (17), 4368-4380.

(3) Katsikogianni, M.; Missirlis, Y. Concise review of mechanisms of bacterial adhesion to biomaterials and of techniques used in estimating bacteria-material interactions. Eur. Cell Mater. 2004, 8, 37-57.

(4) Bos, R.; van der Mei, H. C.; Busscher, H. J. Physico-chemistry of initial microbial adhesive interactions-Its mechanisms and methods for study. FEMS Microbiol. Rev. 1999, 23 (2), 179-230.

(5) Garrett, T. R.; Bhakoo, M.; Zhang, Z. Bacterial adhesion and biofilms on surfaces. Prog. Nat. Sci. 2008, 18 (9), 1049-1056.

(6) Dunne, W. M. Bacterial adhesion: Seen any good biofilms lately? Clin. Microbiol. Rev. 2002, 15 (2), 155-166.

(7) Donlan, R. M. Biofilms: Microbial life on surfaces. Emerging Infect. Dis. 2002, 8 (9), 881-890.

(8) Bruinsma, G. M.; van der Mei, H. C.; Busscher, H. J. Bacterial adhesion to surface hydrophilic and hydrophobic contact lenses. Biomaterials 2001, 22 (24), 3217-3224.

(9) Busscher, H. J.; Rinastiti, M.; Siswomihardjo, W.; van der Mei, H. C. Biofilm formation on dental restorative and implant materials. $J$. Dent. Res. 2010, 89 (7), 657-665.

(10) Ista, L. K.; Callow, M. E.; Finlay, J. A.; Coleman, S. E.; Nolasco, A. C.; Simons, R. H.; Callow, J. A.; Lopez, G. P. Effect of substratum surface chemistry and surface energy on attachment of marine bacteria and algal spores. Appl. Environ. Microbiol. 2004, 70 (7), 4151-4157.

(11) Li, Y.; Gao, Y. H.; Li, X. S.; Yang, J. Y.; Que, G. H. Influence of surface free energy on the adhesion of marine benthic diatom Nitzschia closterium MMDL533. Colloids Surf., B 2010, 75 (2), 550-556.

(12) Logan, B. E.; Regan, J. M. Microbial fuel cells-Challenges and applications. Environ. Sci. Technol. 2006, 40 (17), 5172-5180.

(13) Sharma, P.; Hanumantha Rao, K. Adhesion of Paenibacillus polymyxa on chalcopyrite and pyrite: Surface thermodynamics and extended DLVO theory. Colloids Surf., B 2003, 29 (1), 21-38.

(14) Hermansson, M. The DLVO theory in microbial adhesion. Colloids Surf., B 1999, 14 (1), 105-119.

(15) Van der Mei, H.; Bos, R.; Busscher, H. A reference guide to microbial cell surface hydrophobicity based on contact angles. Colloids Surf., B 1998, 11 (4), 213-221.

(16) van Loosdrecht, M. C.; Lyklema, J.; Norde, W.; Schraa, G.; Zehnder, A. J. The role of bacterial cell wall hydrophobicity in adhesion. Appl. Environ. Microbiol. 1987, 53 (8), 1893-1897.

(17) Busscher, H. J.; Weerkamp, A. H.; van der Mei, H. C.; van Pelt, A. W.; de Jong, H. P.; Arends, J. Measurement of the surface free energy of bacterial cell surfaces and its relevance for adhesion. Appl. Environ. Microbiol. 1984, 48 (5), 980-983.

(18) Li, B.; Logan, B. E. Bacterial adhesion to glass and metal-oxide surfaces. Colloids Surf., B 2004, 36 (2), 81-90.
(19) Briandet, R.; Meylheuc, T.; Maher, C.; Bellon-Fontaine, M. N. Listeria monocytogenes Scott A: Cell surface charge, hydrophobicity, and electron donor and acceptor characteristics under different environmental growth conditions. Appl. Environ. Microbiol. 1999, 65 (12), 5328-5333.

(20) Azeredo, J.; Visser, J.; Oliveira, R. Exopolymers in bacterial adhesion: Interpretation in terms of DLVO and XDLVO theories. Colloids Surf., B 1999, 14 (1), 141-148.

(21) Becker, K. Exopolysaccharide production and attachment strength of bacteria and diatoms on substrates with different surface tensions. Microb. Ecol. 1996, 32 (1), 23-33.

(22) Busscher, H. J.; Van Der Mei, H. C. Physico-chemical interactions in initial microbial adhesion and relevance for biofilm formation. Adv. Dent. Res. 1997, 11 (1), 24-32.

(23) Genzer, J.; Efimenko, K. Recent developments in superhydrophobic surfaces and their relevance to marine fouling: A review. Biofouling 2006, 22 (5-6), 339-360.

(24) Hallab, N. J.; Bundy, K. J.; O’Connor, K.; Moses, R. L.; Jacobs, J. J. Evaluation of metallic and polymeric biomaterial surface energy and surface roughness characteristics for directed cell adhesion. Tissue Eng. 2001, 7 (1), 55-71.

(25) Allion, A.; Baron, J.-P.; Boulange-Petermann, L. Impact of surface energy and roughness on cell distribution and viability. Biofouling 2006, 22 (5), 269-278.

(26) Loo, C.-Y.; Young, P. M.; Lee, W.-H.; Cavaliere, R.; Whitchurch, C. B.; Rohanizadeh, R. Superhydrophobic, nanotextured polyvinyl chloride films for delaying Pseudomonas aeruginosa attachment to intubation tubes and medical plastics. Acta Biomater. 2012, 8 (5), $1881-1890$.

(27) Teughels, W.; Van Assche, N.; Sliepen, I.; Quirynen, M. Effect of material characteristics and/or surface topography on biofilm development. Clin. Oral Implants Res. 2006, 17 (S2), 68-81.

(28) Vadillo-Rodriguez, V.; Logan, B. E. Localized attraction correlates with bacterial adhesion to glass and metal oxide substrata. Environ. Sci. Technol. 2006, 40 (9), 2983-2988.

(29) Absolom, D. R.; Lamberti, F. V.; Policova, Z.; Zingg, W.; van Oss, C. J.; Neumann, A. Surface thermodynamics of bacterial adhesion. Appl. Environ. Microbiol. 1983, 46 (1), 90-97.

(30) Park, B.-J.; Abu-Lail, N. I. The role of the $\mathrm{pH}$ conditions of growth on the bioadhesion of individual and lawns of pathogenic Listeria monocytogenes cells. J. Colloid Interface Sci. 2011, 358 (2), 611620 .

(31) Morisaki, H.; Tabuchi, H. Bacterial attachment over a wide range of ionic strengths. Colloids Surf., B 2009, 74 (1), 51-55.

(32) Bayoudh, S.; Othmane, A.; Mora, L.; Ben Ouada, H. Assessing bacterial adhesion using DLVO and XDLVO theories and the jet impingement technique. Colloids Surf., B 2009, 73 (1), 1-9.

(33) Katsikogianni, M.; Missirlis, Y. Interactions of bacteria with specific biomaterial surface chemistries under flow conditions. Acta Biomater. 2010, 6 (3), 1107-1118.

(34) Vilinska, A.; Rao, K. H. Surface thermodynamics and extended DLVO theory of Acidithiobacillus ferrooxidans cells adhesion on pyrite and chalcopyrite. Open Colloid Sci. J. 2009, 2, 1-14.

(35) Jucker, B. A.; Zehnder, A. J. B.; Harms, H. Quantification of polymer interactions in bacterial adhesion. Environ. Sci. Technol. 1998, 32 (19), 2909-2915.

(36) Morra, M.; Cassinelli, C. Bacterial adhesion to polymer surfaces: A critical review of surface thermodynamic approaches. J. Biomater. Sci., Polym. Ed. 1998, 9 (1), 55-74.

(37) van Loosdrecht, M. C.; Lyklema, J.; Norde, W.; Zehnder, A. J. Bacterial adhesion: A physicochemical approach. Microb. Ecol. 1989, 17 (1), 1-15.

(38) Nguyen, V. T.; Chia, T. W. R.; Turner, M. S.; Fegan, N.; Dykes, G. A. Quantification of acid-base interactions based on contact angle measurement allows XDLVO predictions to attachment of Campylobacter jejuni but not Salmonella. J. Microbiol. Methods 2011, 86 (1), $89-96$. 
(39) Sharma, P. K.; Rao, K. H. Analysis of different approaches for evaluation of surface energy of microbial cells by contact angle goniometry. Adv. Colloid Interface Sci. 2002, 98 (3), 341-463.

(40) Zuo, Y.; Li, D.; Neumann, A. W. Wettability and surface tension of particles. In Applied Surface Thermodynamics, 2nd ed.; Neumann, A. W., Robert, D., Zuo, Y., Eds.; CRC Press: Boca Raton, FL, 2010; p 599.

(41) Zhang, X.; Jiang, Z.; Li, M.; Zhang, X.; Wang, G.; Chou, A.; Chen, L.; Yan, H.; Zuo, Y. Y. Rapid spectrophotometric method for determining surface free energy of microalgal cells. Anal. Chem. 2014, 86 (17), 8751-8756.

(42) Zhang, X.; Zhang, Q.; Yan, T.; Jiang, Z.; Zhang, X.; Zuo, Y. Y. Surface free energy activated high-throughput cell sorting. Anal. Chem. 2014, 86 (18), 9350-9355.

(43) Kwok, D. Y.; Neumann, A. W. Contact angle measurement and contact angle interpretation. Adv. Colloid Interface Sci. 1999, 81 (3), 167-249.

(44) Zuo, Y. Y.; Ding, M.; Bateni, A.; Hoorfar, M.; Neumann, A. W. Improvement of interfacial tension measurement using a captive bubble in conjunction with axisymmetric drop shape analysis (ADSA). Colloids Surf., A 2004, 250 (1-3), 233-246.

(45) Rafiee, J.; Mi, X.; Gullapalli, H.; Thomas, A. V.; Yavari, F.; Shi, Y.; Ajayan, P. M.; Koratkar, N. A. Wetting transparency of graphene. Nat. Mater. 2012, 11, 217-222.

(46) O’Toole, G.; Kaplan, H. B.; Kolter, R. Biofilm formation as microbial development. Annu. Rev. Microbiol. 2000, 54, 49-79.

(47) Krasowska, A.; Sigler, K. How microorganisms use hydrophobicity and what does this mean for human needs? Front. Cell. Infect. Microbiol. 2014, 4, 112.

(48) Rosenberg, M. Microbial adhesion to hydrocarbons: Twentyfive years of doing MATH. FEMS Microbiol. Lett. 2006, 262 (2), 129134.

(49) Ruhs, P. A.; Bocker, L.; Inglis, R. F.; Fischer, P. Studying bacterial hydrophobicity and biofilm formation at liquid-liquid interfaces through interfacial rheology and pendant drop tensiometry. Colloids Surf., B 2014, 117, 174-184.

(50) Chmielewski, R. A. N.; Frank, J. F. Biofilm formation and control in food processing facilities. Compr. Rev. Food Sci. Food Saf. 2003, 2 (1), 22-32.

(51) Donlan, R. M. Biofilm formation: A clinically relevant microbiological process. Clin. Infect. Dis. 2001, 33 (8), 1387-1392. 